\title{
Relationship between intraocular pressure and retinal nerve fibre thickness loss in a monkey model of chronic ocular hypertension
}

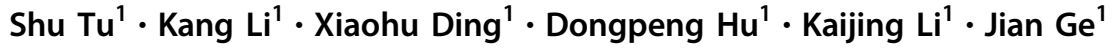

Received: 22 August 2018 / Revised: 31 January 2019 / Accepted: 27 April 2019 / Published online: 21 June 2019

(c) The Author(s), under exclusive licence to The Royal College of Ophthalmologists 2019

\begin{abstract}
Chronic ocular hypertension (COHT) monkey models were established by destroying the trabecular meshwork, for investigating the relationship between intraocular pressure (IOP) and retinal nerve fibre layer (RNFL) thickness loss. IOP and RNFL thickness were measured before laser injury and weekly thereafter for 27 weeks using Tono Vet and Stratus optical coherence tomography (OCT). The quantitative relationship was as follows: (1) at 32-47 $\mathrm{mmHg}$, the average damage rate was $-3.08 \pm 0.28 \mu \mathrm{m} /$ week; (2) at $25-30 \mathrm{mmHg}$, it was $-1.45 \pm 0.19 \mu \mathrm{m} /$ week. The inferior RNFL and superior RNFL turned out to be the most IOP-sensitive quadrants with the rate of RNFL change almost in parallel with IOP levels. The superior sector seemed to be resistant to high IOP conditions until a RNFL loss of $\sim 20 \mu \mathrm{m}$ was detected in the inferior sector. The rate of RNFL thickness loss was slowed with obvious turning points at RNFL thicknesses of $\sim 75 \mu \mathrm{m}, 65 \mu \mathrm{m}$, and $50 \mu \mathrm{m}$. The experimental results have achieved research significance. The COHT Monkey was an ideal animal model that can be used for evaluating the relationship between IOP and RNFL damage. Higher IOP was associated with faster RNFL thickness loss. The level of IOP was a vital factor for RNFL damage rate, and baseline/residual RNFL thickness was also important for subsequent RNFL damage. OCT was suitable for measuring RNFL thickness change in COHT monkey models.
\end{abstract}

\section{Introduction}

Intraocular pressure (IOP) is a major risk factor for the development of glaucoma and its progression [1,2]. Higher IOP leads to more serious RNFL thickness loss and worsening of the visual field. The European Glaucoma Society (EGS) has suggested that lowering IOP is the only approach confirmed to be efficient for preserving visual function [3]. The Ocular Hypertension Treatment Study (OHTS) and the Advanced Glaucoma Intervention Study (AGIS) have proved the role of IOP in glaucoma, as well as the benefits of IOP-lowering treatment [4-6]. The Early Manifest Glaucoma Trial (EMGT) demonstrated that progression risk decreased by $\sim 10 \%$ of IOP reduction from baseline to the first follow-up visit [7]. In recent years, some clinical trials considered that baseline retinal nerve fibre layer (RNFL)

Jian Ge

gejian@mail.sysu.edu.cn

1 State Key Laboratory of Ophthalmology, Guangdong Provincial Key Laboratory of Ophthalmology and Visual Science, Zhongshan Ophthalmic Center, Sun Yat-sen University, Guangzhou, China thickness can affect the rate of RNFL thinning. A thicker baseline RNFL was associated with an increased rate in the reduction of RNFL thickness [8, 9]. Several baseline RNFL and ganglion cell complex (GCC) parameters were found to be significant predictors of progression, and an abnormal GCC focal loss volume at baseline increased the risk of progression by a hazard ratio of 3.1 [10].

However, there is a lack of evidence about the dynamic relationship between the impact of IOP and changes in RNFL thickness because of medical ethics. How baseline/ residual RNFL thickness influences RNFL thinning has yet to be studied. More animal research has been conducted to investigate the relationship between elevated IOP and RNFL thickness changes, and some advancements were made [11-13]. However, the quantitative relationship between different levels of IOPs and RNFL thickness loss needs to be explored. Moreover, other problems, such as the effect of baseline/residual RNFL thickness on subsequent RNFL thickness damage and how the 24-h IOP fluctuation affects RNFL thickness loss, also need to be investigated.

In this study, a chronic ocular hypertension (COHT) monkey model was also successfully established through destroying trabecular meshwork with laser photocoagulation 
according to a method from our previous study. Baseline IOP, RNFL thickness, optic nerve head parameters, and the RNFL damage principle of "ISNT" were reported in our published work [14]. Such models highly mimic the status of open angle glaucoma with IOP being elevated chronically and gradually. Optical coherence tomography (OCT) has been demonstrated to be capable of accurately detecting RNFL thickness in monkey models with experimentally induced glaucoma [15-17]. Longitudinal OCT scanning would provide a better understanding of that relationship in a glaucoma monkey model. This study focused on OCT measurements of RNFL thickness changes associated with different levels of IOP to obtain a dynamic longitudinal observation in 27 weeks in the stable primate COHT model without drug intervention, thus trying to reveal the glaucoma progression pattern.

\section{Materials and methods}

\section{Animals and anaesthesia}

The study procedure was performed according to the ARVO Statement for the Use of Animals in Ophthalmic and Vision Research. Thirteen adult rhesus monkeys (4-year-old to 6-year-old males weighing between 4 and $8 \mathrm{~kg}$ ) were obtained from Landao Biotechnology Co., Ltd. (Guangdong, China) and used for investigating the relationship between high IOP and RNFL damage. Monkeys were raised in large cages with adequate room for activities and were fed nutritious food and water. The rooms were illuminated with a 12-h light-dark cycle (with a daytime light intensity of 200 lux) under controlled humidity $(50-55 \%)$ and temperature $\left(24-25^{\circ} \mathrm{C}\right)$ conditions. The monkeys' health was monitored daily by animal care staff and veterinary personnel. All experimental procedures were performed under deep general anaesthesia via an intramuscular injection of ketamine hydrochloride $\left(5 \mathrm{mg} / \mathrm{kg}\right.$, Ketalar $50^{\circledR}$, GuTian Pharmaceuticals Ltd, Fujian, China) plus chlorpromazine hydrochloride $\left(2.5 \mathrm{mg} / \mathrm{kg}\right.$, chlorpromazine $50^{\circledR}$, JiaoZuo Pharmaceuticals Ltd, Tianjin, China). Topical corneal anaesthesia was provided with $0.5 \%$ proparacaine eye drops (Alcaine ${ }^{\circledast}$, Alcon Laboratories Inc.).

\section{Establishing the chronic ocular hypertension monkey model}

Thirteen right eyes of 13 monkeys were planned to be used for the COHT monkey model. All the animals underwent a regular ophthalmological examination, including IOP measurement, slit-lamp biomicroscopy, corneal pachymetry, optic disc photography, and OCT measurement of RNFL thickness prior to laser photocoagulation to exclude any existing ocular diseases and obtain baseline ophthalmic data. Pupils were sufficiently contracted $(1 \mathrm{~mm})$ with $1 \%$ pilocarpine eye drops (Pilocarpine ${ }^{\circledR}$; Zhongshan ophthalmic center, Guangdong, China). The entire circumference of the trabecular meshwork (TM) was ablated with a VISULAS Trion laser (VISULAS Trion; Carl Zeiss Meditec AG, Goeschwitzer Strasse, Jena, USA) using the silt lamp delivery system and a laser gonioscope, as previously described with some modifications $[18,19]$. The laser parameters were as follows: $50 \mu \mathrm{m}$ spot size, 0.5 -s duration, 800-1200 mw laser power and 150-250 spots. Care was taken to photocoagulate the middle TM. Presence of a vapour bubble signified an effective ablation in the TM. Tobramycin dexamethasone and Tropicamide eye drops (Alcon Laboratories Inc.) were used for alleviating the noninfectious inflammation during the immediate post-laser photocoagulation period. If the IOP was not consistently higher than $21 \mathrm{mmHg}$, additional laser photocoagulation was performed again in intervals of three weeks until stable intraocular hypertension was obtained. Narrowing of the neuroretinal rim, thinning of the RNFL thickness and corresponding elevated IOP were monitored weekly after the first laser treatment to define the COHT model.

\section{Central corneal thickness (CCT) assessment}

Anterior segment optical coherence tomography (AS-OCT; Fourier OCT, Carl Zeiss Meditec, Dublin CA, USA) was used for measuring CCT before the first laser photocoagulation to exclude the effect of corneal thickness of the animals themselves on IOP measurement. A procedure with the four lines scan model of the cornea was applied. The line is centred on the corneal vertex, and the procedure is performed with horizontal, vertical, $45^{\circ}$ and $135^{\circ}$ scans. The animals' eye positions and the scan lines were especially noticeable through the central cornea.

\section{Measurement of intraocular pressure}

IOP was measured using the Tono $\mathrm{Vet}^{\circledR}$ rebound tonometer (Finland, TV01) according to the manufacturers' recommended procedures. Animals without a topical eye anaesthetic were seated and held gently at a desk by one operator, then another operator measured the IOP. The tonometer was corrected before each measurement. Six consecutive IOP readings were obtained, and the average value was automatically calculated. Only consecutive readings with little deviation $(<3 \mathrm{mmHg})$ were regarded as valid measurements. The measurement was repeated three times (for a total of 18 separate measurements) for the average value, which was applied in the statistical analyses. IOPs were measured weekly, and only measurements taken on the same day of the OCT RNFL scans were included in the study. 


\section{Optic disc photography}

Colour and white fundus photographs with a $35^{\circ}$ angle were collected from anaesthetised animals using a retinal camera (TRC-50DX RETINAL CAMERA; Topcon, Tokyo, Japan) and a Nikon $200 \mathrm{D}$ digital camera. The pupils were dilated $(8 \mathrm{~mm})$ with $0.25 \%$ Tropicamide eye drops (Mydrin ${ }^{\circledR}$, Santen Pharmaceutical, Osaka, Japan), and an ocular lubricating agent (Artificial tears ${ }^{\circledR}$; Zhongshan ophthalmic center, Guangdong, China) was applied to preserve corneal clarity during the examination. The narrowed neuroretinal rim and the enlarged optic cup were observed in the animal model.

\section{OCT measurements of RNFL thickness}

STRATUS OCT (Model 3000, Carl Zeiss Meditec, Dublin CA, USA) was used to measure peripapillary RNFL thickness prior to the laser treatment, and then measurements were made weekly after the first laser ablation. Crosssectional imaging of RNFL was obtained in a circle scan (3.4 mm diameter). Similar to optic disc photography, the animals' pupils were dilated sufficiently, and the corneas were kept clear during the examination. All the scans were centred on the optic disc by changing the animals' head positions. The RNFL thickness was calculated in the analysis printout after averaging the results of three sequential circular scans. Only OCT images obtained at a good signal strength (signal strength $\geq 7$ ) were selected for analysis. The thinned RNFL thickness was examined in the animal model.

\section{Grouping based on RNFL thickness loss}

Similar to humans, the physiological average RNFL thickness of monkeys was $\sim 100 \mu \mathrm{m}$. This study divided all COHT monkey models into two equal groups (6 in each group) based on the final residual RNFL thickness (MEDIAN: $54 \mu \mathrm{m})$.

\section{Statistical analysis}

This was a longitudinal observational study about the relationship between IOP and RNFL damage. Data in the RNFL thickness variation tendency graph were expressed as the means \pm SEM. $P<0.05$ was considered statistically significant in all the analyses. A regression model of RNFL thickness changes was fitted with fixed coefficients (fixed effects), which was used to dynamically evaluate the relationship between average IOP and RNFL thickness loss over time while considering central corneal thickness, acute high IOP, and baseline/residual RNFL thickness. OCT measurements of RNFL thickness were considered to be the dependent variable, while time and baseline/residual RNFL thickness were included as continuous predictors. Statistical analyses were performed using SPSS software (Version 13.0, SPSS, Chicago, IL, USA).

\section{Results}

Twelve COHT monkey models (right eyes) were successfully established, one failed, and the success rate was $92.31 \%$ (Fig. 1a). The average CCT of the 12 animal models was $489.17 \pm 20.65 \mu \mathrm{m}$ (mean $\pm \mathrm{SD}$ ). The correlation coefficient $\left(r^{2}\right)$ between IOP and CCT was $-0.37(P=$ 0.236 ). IOP measurements were not significantly influenced by CCT in this study. Table 1 presents IOP and RNFL thickness loss pre-laser and post-laser photocoagulation. There was no statistical difference in the IOP/RNFL thickness of both groups before modelling $(P>0.05)$. Figure $1 \mathrm{~b}$ displays the morphological change of RNFL thickness.

The 12 monkey models were divided into two groups based on the final residual RNFL thickness. Several laser photocoagulations were performed on each animal model throughout the experiment. Each laser treatment usually maintained high IOP for 3 to 6 weeks [14]. The level and duration of high IOP in the both groups varied and led to different damage rates of the RNFL. In group A, IOP was sustained between $25 \mathrm{mmHg}$ and $30 \mathrm{mmHg}$, the rate of global average RNFL thickness change was $-2.66 \pm 0.44$ $\mu \mathrm{m} /$ week (CI: -1.84 to $-1.06 \mu \mathrm{m} /$ week; $P=0.000$ ) before the 14th week and $-0.28 \pm 0.12 \mu \mathrm{m} /$ week (CI: -0.51 to $-0.44 \mu \mathrm{m} /$ week; $P=0.022$ ) thereafter. In group $\mathrm{B}$, IOP sharply increased to $32-47 \mathrm{mmHg}$ in the first 14 weeks, with the corresponding rate being $-3.08 \pm 0.28 \mu \mathrm{m} /$ week (CI: -3.66 to $-2.51 \mu \mathrm{m} /$ week; $P=0.000$ ), whereas for the next 13 weeks, the rate decreased to $-0.79 \pm 0.14 \mu \mathrm{m} /$ week (CI: -1.07 to $-0.52 \mu \mathrm{m} /$ week; $P=0.000$ ). Figures 2,3 and Tables 2, 3 illustrate the relationship between IOP and slopes of RNFL loss for the global average and sectorial RNFL thickness parameters. The higher IOP could lead to a quicker RNFL damage rate. The inferior RNFL (INFRNFL) and superior RNFL (SUP-RNFL) turned out to be the most IOP-sensitive quadrants, with the rate of RNFL change occurring almost in parallel with IOP levels. The temporal superior sector seemed to be resistant to high IOP conditions until a RNFL loss of $\sim 20 \mu \mathrm{m}$ was detected in the temporal inferior sector. As demonstrated in a clinical setting, the nasal RNFL (NAS-RNFL) and the temporal RNFL (TEMP-RNFL) displayed unstable change in COHT monkey models.

Another interesting phenomenon was investigated. Seven of the 12 monkey models had IOP that became rapidly elevated to $40-50 \mathrm{mmHg}$ after the first photocoagulation, 
Fig. 1 A monkey model of chronic ocular hypertension was established. a Chronic intraocular hypertension monkey model with an enlarged optic cup (black arrows) and a damaged retinal nerve fibre layer (asterisk); b Different degrees of damage in the retinal nerve fibre layer in a chronic intraocular hypertension monkey model
Table 1 Intraocular pressure (IOP) and different quadrants of retinal nerve fibre layer (RNFL) thickness variation pre-laser and post-laser treatment

\section{A}

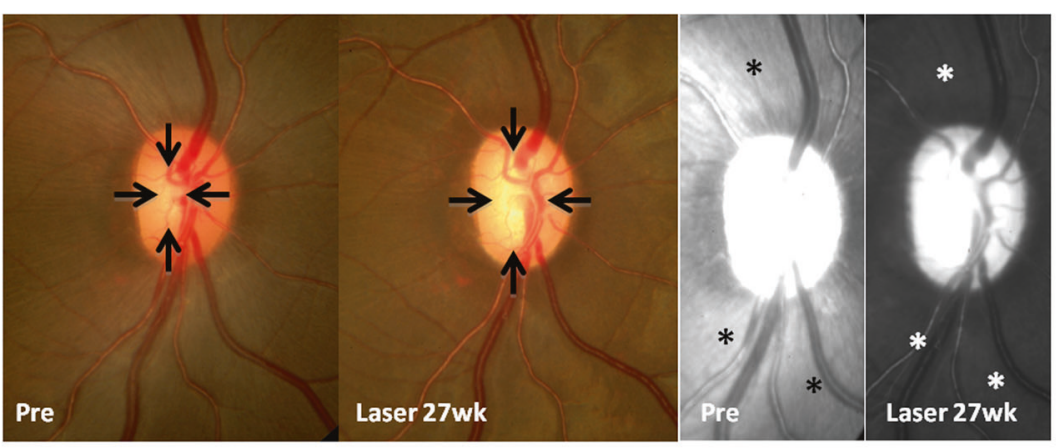

B

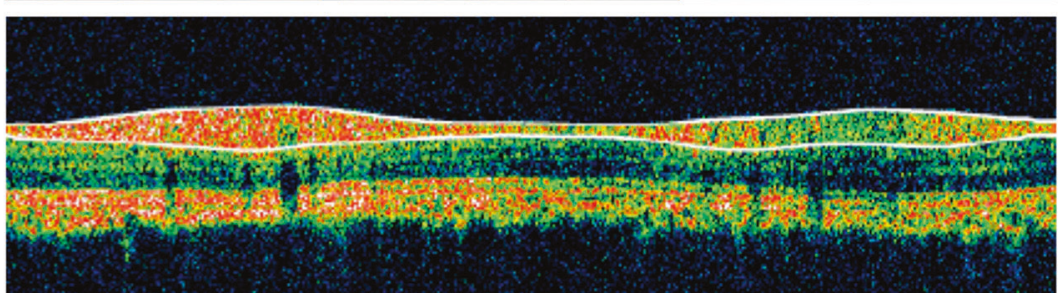

Pre, AVG-RNFLT: $99 \mu \mathrm{m}$

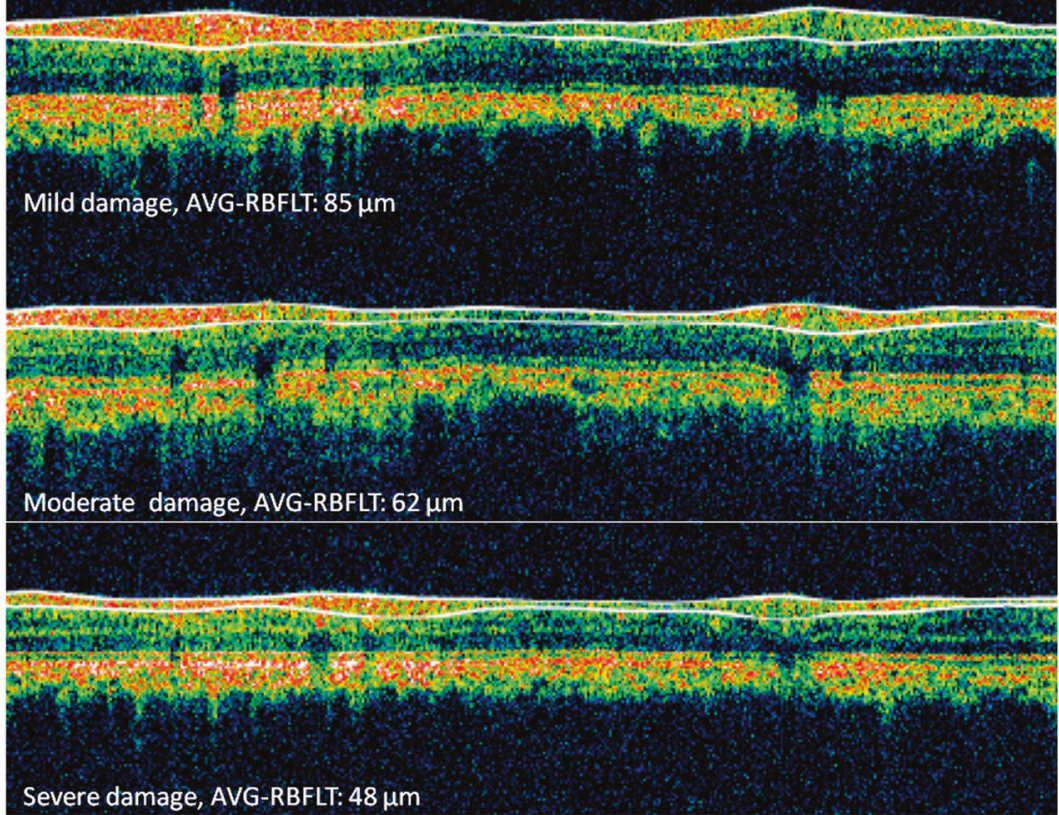

\begin{tabular}{lcllll}
\hline \multirow{2}{*}{$\begin{array}{l}\text { Group } \\
\text { Time }\end{array}$} & \multicolumn{2}{l}{ Group A (six eyes) } & & \multicolumn{2}{l}{ Group B (six eyes) } \\
\cline { 2 - 3 } \cline { 6 - 6 } \cline { 5 - 6 } & Pre & The 27th week & & Pre & The 27th week \\
\hline IOP $(\mathrm{mmHg})$ & $15.83 \pm 1.17$ & $19.83 \pm 4.96$ & & $14.00 \pm 2.61$ & $24.33 \pm 14.12$ \\
Average $(\mu \mathrm{m})$ & $100.33 \pm 9.05$ & $73.83 \pm 12.62$ & & $96.00 \pm 4.15$ & $43.00 \pm 2.97$ \\
Inferior $(\mu \mathrm{m})$ & $142.83 \pm 15.82$ & $85.33 \pm 19.71$ & & $138.50 \pm 11.54$ & $46.83 \pm 6.79$ \\
Superior $(\mu \mathrm{m})$ & $118.50 \pm 13.85$ & $98.00 \pm 18.92$ & & $115.67 \pm 9.24$ & $52.00 \pm 4.56$ \\
Nasal $(\mu \mathrm{m})$ & $66.67 \pm 12.23$ & $55.17 \pm 12.56$ & & $64.50 \pm 8.87$ & $32.33 \pm 3.33$ \\
Temporal $(\mu \mathrm{m})$ & $73.00 \pm 5.80$ & $58.67 \pm 9.11$ & & $66.00 \pm 8.65$ & $39.83 \pm 6.24$ \\
\hline
\end{tabular}

Note: Data are expressed as the mean $\pm \mathrm{SD}$ 

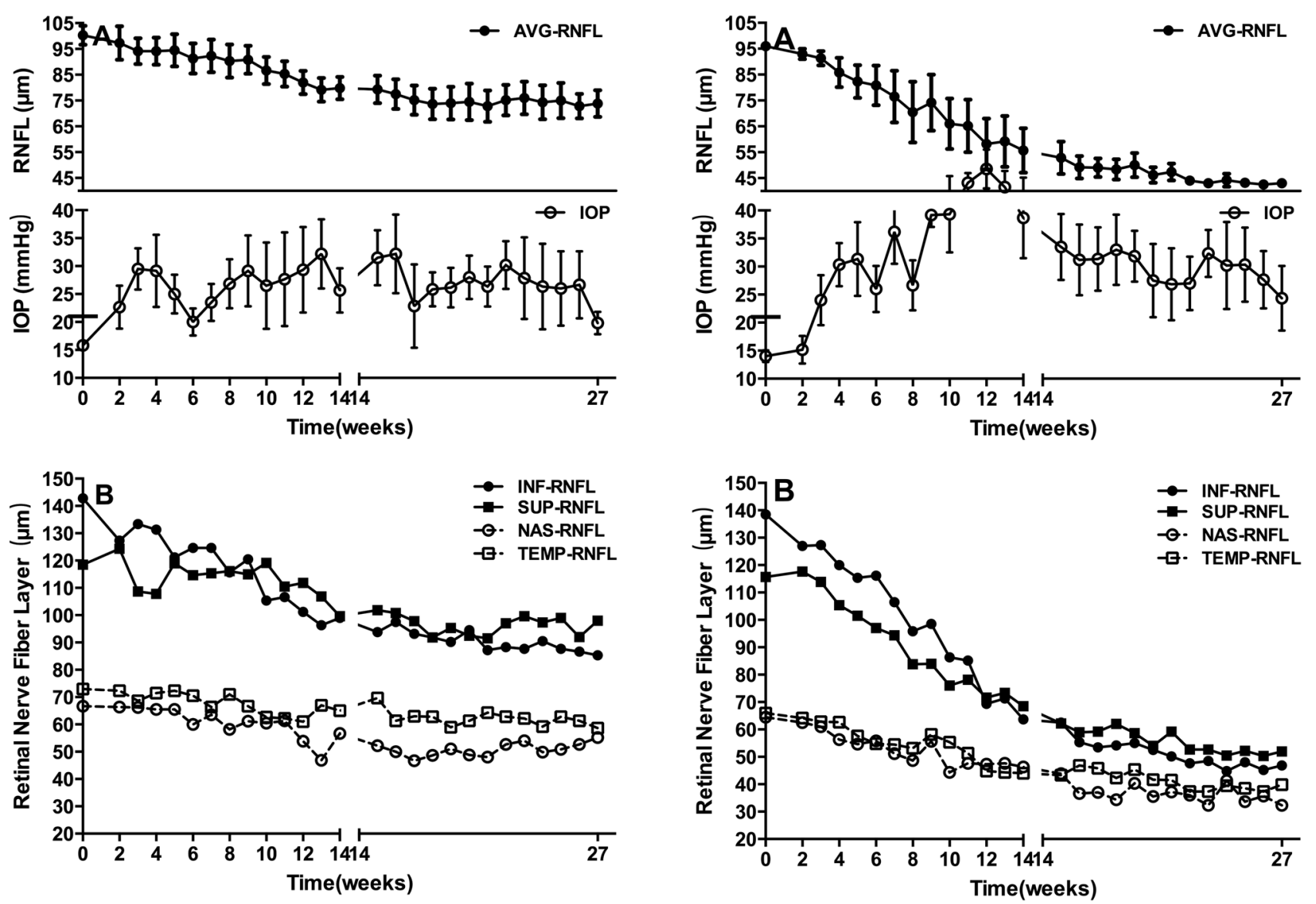

Fig. 2 The relationship between intraocular pressure (IOP) and retinal nerve fibre layer (RNFL) thickness in group A. a The damage trend in the average RNFL thickness associated with the elevated IOP; $\mathbf{b}$ The damage trend of RNFL thickness in the inferior (INF), superior (SUP), nasal (NAS), and temporal (TEMP) quadrants

and the average RNFL thickness was sharply decreased from $97 \mu \mathrm{m}$ to $75 \mu \mathrm{m}$ at a rate of $-21.86 \pm 3.10 \mu \mathrm{m} /$ week (CI: -29.45 to $-14.26 ; P=0.000)$. In the following six weeks, IOPs were still kept $\sim 40 \mathrm{mmHg}$, and the average RNFL thickness dropped from $75 \mu \mathrm{m}$ to $65 \mu \mathrm{m}$ at a rate of $-4.00 \pm 1.70 \mu \mathrm{m} /$ week (CI: -8.73 to $0.73 ; P=0.000$ ), then decreased to $50 \mu \mathrm{m}$ at a rate of $-2.56 \pm 2.01 \mu \mathrm{m} /$ week (CI: -7.49 to $2.37 ; P=0.251$ ) (Fig. 4 ). It can be seen that an average RNFL thickness of $\sim 75 \mu \mathrm{m}$ appeared to be the first turning point in a damage rate, then it was $\sim 65 \mu \mathrm{m}$ and followed by $50 \mu \mathrm{m}$. The level of IOP was one of the most vital factors for RNFL damage rate, and the baseline/residual RNFL thickness was also important for subsequent RNFL damage.

\section{Discussion}

This study focused on RNFL thickness change over 27 weeks in COHT monkey models. The animal model
Fig. 3 The relationship between intraocular pressure (IOP) and retinal nerve fibre layer (RNFL) thickness in group B. a The damage trend in the average RNFL thickness associated with the elevated IOP; $\mathbf{b}$ The damage trend of RNFL thickness in the inferior (INF), superior (SUP), nasal (NAS), and temporal (TEMP) quadrants

closely mimicked the pathology of open angle glaucoma. The relationship between the levels of IOP and RNFL thickness loss without drug intervention was dynamically observed in these models.

The purpose of this study was to investigate the relationship between IOP and RNFL thickness loss in a COHT monkey model. Since the eye structure, the IOP, and the RNFL thickness of the rhesus monkey are very close to the human eye, and the COHT monkey models that received laser photocoagulation treatment to destroy the drainage function of trabecular meshwork could resemble open-angle glaucoma, which also occurs following trabecular meshwork dysfunction or degeneration. Although other glaucoma animal models, such as rats, are usually established by injecting BBS to the anterior chamber or cauterisation of the superior scleral vein to increase IOP, they can seldom simulate the pathogenesis of POAG. In addition, the baseline IOP and RNFL of rat models are quite different from humans [20, 21]. In addition, from some publications and our previous research, baseline IOP, baseline RNFL 
Table 2 The damage rate of the retinal fibre layer (RNFL) thickness in group A

\begin{tabular}{|c|c|c|c|c|c|c|}
\hline \multirow[t]{2}{*}{ Quadrant } & \multirow[t]{2}{*}{ Time (after laser/L) } & \multicolumn{2}{|c|}{$\begin{array}{l}\text { RNFL } \\
\text { thickness }(\mu \mathrm{m})\end{array}$} & \multirow[t]{2}{*}{$\begin{array}{l}\text { Reduction rate of RNFL } \\
\text { thickness }(\mu \mathrm{m} / \text { week })\end{array}$} & \multirow[t]{2}{*}{$95 \% \mathrm{CI}$} & \multirow[t]{2}{*}{$P$} \\
\hline & & Pre & Post & & & \\
\hline \multirow[t]{2}{*}{ Average } & Pre-L14 week & 100 & 80 & $-1.45 \pm 0.19$ & $-1.84,-1.06$ & 0.000 \\
\hline & L 15 week-L 27 week & 80 & 74 & $-0.28 \pm 0.12$ & $-0.51,-0.04$ & 0.022 \\
\hline \multirow[t]{2}{*}{ Inferior } & Pre-L14week & 143 & 99 & $-3.16 \pm 0.36$ & $-3.88,-2.43$ & 0.000 \\
\hline & L 15 week-L 27 week & 94 & 85 & $-0.78 \pm 0.23$ & $-1.24,-0.32$ & 0.001 \\
\hline \multirow[t]{2}{*}{ Superior } & Pre-L14 week & 119 & 100 & $-0.82 \pm 0.36$ & $-1.54,-0.10$ & 0.026 \\
\hline & L 15 week-L 27 week & 102 & 98 & $-0.18 \pm 0.24$ & $-0.65,0.29$ & 0.448 \\
\hline \multirow[t]{2}{*}{ Nasal } & Pre-L14 week & 67 & 57 & $-1.08 \pm 0.27$ & $-1.62,-0.55$ & 0.000 \\
\hline & L 15 week-L 27 week & 52 & 55 & $0.34 \pm 0.20$ & $-0.06,0.73$ & 0.094 \\
\hline \multirow[t]{2}{*}{ Temporal } & Pre-L14 week & 73 & 65 & $-0.75 \pm 0.25$ & $-1.24,-0.26$ & 0.003 \\
\hline & L 15 week-L 27 week & 70 & 59 & $-0.38 \pm 0.26$ & $-0.91,0.14$ & 0.147 \\
\hline
\end{tabular}

Note: Data are expressed as the mean \pm SEM

Table 3 The damage rate of retinal fibre layer (RNFL) thickness in group B

\begin{tabular}{|c|c|c|c|c|c|c|}
\hline \multirow[t]{2}{*}{ Quadrant } & \multirow[t]{2}{*}{ Time (after laser/L) } & \multicolumn{2}{|c|}{$\begin{array}{l}\text { RNFL } \\
\text { thickness }(\mu \mathrm{m})\end{array}$} & \multirow{2}{*}{$\begin{array}{l}\text { Reduction rate of } \\
\text { RNFL thickness } \\
(\mu \mathrm{m} / \text { week) }\end{array}$} & \multirow[t]{2}{*}{$95 \% \mathrm{CI}$} & \multirow[t]{2}{*}{$P$} \\
\hline & & Pre & Post & & & \\
\hline \multirow[t]{2}{*}{ Average } & Pre-L 14 week & 96 & 56 & $-3.08 \pm 0.28$ & $-3.66,-2.51$ & 0.000 \\
\hline & L 15 week-L 27 week & 53 & 43 & $-0.79 \pm 0.14$ & $-1.07,-0.52$ & 0.000 \\
\hline \multirow[t]{2}{*}{ Inferior } & Pre-L14 week & 139 & 64 & $-5.45 \pm 0.48$ & $-6.41,-4.50$ & 0.000 \\
\hline & L 15 week-L 27 week & 63 & 47 & $-1.17 \pm 0.24$ & $-1.65,-0.68$ & 0.000 \\
\hline \multirow[t]{2}{*}{ Superior } & Pre-L14 week & 116 & 69 & $-3.92 \pm 0.40$ & $-4.72,-3.12$ & 0.000 \\
\hline & L 15 week-L 27 week & 62 & 52 & $-0.99 \pm 0.23$ & $-1.45,-0.53$ & 0.000 \\
\hline \multirow[t]{2}{*}{ Nasal } & Pre-L14 week & 65 & 46 & $-1.37 \pm 0.27$ & $-1.90,-0.84$ & 0.000 \\
\hline & L 15 week-L 27 week & 44 & 32 & $-0.45 \pm 0.22$ & $-0.90,-0.00$ & 0.049 \\
\hline \multirow[t]{2}{*}{ Temporal } & Pre-L14 week & 66 & 44 & $-1.59 \pm 0.26$ & $-2.11,-1.07$ & 0.000 \\
\hline & L 15 week-L 27 week & 43 & 40 & $-0.70 \pm 0.20$ & $-1.09,-0.30$ & 0.001 \\
\hline
\end{tabular}

Note: Data are expressed as the mean \pm SEM

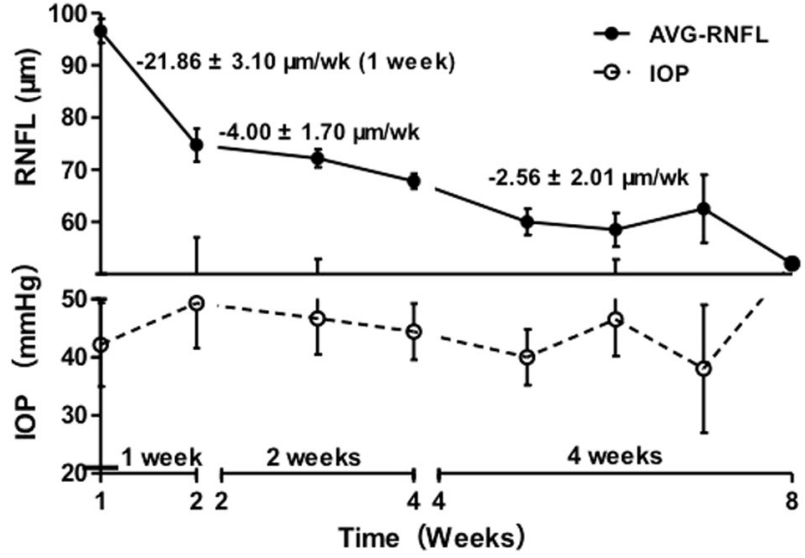

Fig. 4 The relationship between baseline/residual retinal nerve fibre layer thickness (RNFL) and the rate of subsequent RNFL thickness loss thickness, optic nerve head parameters, and the RNFL damage principle of "ISNT" in monkeys have been well characterised [13-15, 22]. Though every monkey experienced several laser treatments and repeated high IOP in this study, IOP was chronically and gradually elevated without sharp increases or human regulation. It was suggested that IOP was closely related to RNFL thickness loss. After laser treatment, the monkeys' IOPs were increased to different levels, and RNFL thickness displayed different damage rates. The higher IOP could lead to a quicker RNFL damage rate. The RNFL thickness loss rate slowed down when IOP gradually decreased. These findings, which were similar to open angle glaucoma, demonstrated that the COHT monkey model was an ideal animal model that can be used for evaluating the relationship between IOP and RNFL thickness damage. 
Allberto Diniz-Filho reported that $1 \mathrm{mmHg}$ higher average IOP was associated with an additional average loss of $0.20 \mu \mathrm{m} /$ year of global RNFL thickness versus $0.04 \mu \mathrm{m} /$ year for nonprogressing eyes [23]. Christopher Bowd showed that the global change in moderate-to-advanced glaucoma was $-0.36 \mu \mathrm{m} /$ year circumpapillary RNFL thickness [24]. These studies were helpful for evaluating RNFL damage risk. Although, the rate of global RNFL loss associated with each $1 \mathrm{mmHg}$ increase in average IOP was not calculated in this study. The rate of RNFL loss for different levels of IOP was obtained in monkey models. It was observed that IOP above $35 \mathrm{mmHg}$ was dangerous for RNFL thickness loss. The experimental results have a certain clinical significance. In clinical practice, some patients (with poor eyesight and a severely damaged optic nerve) might require several operations combined along with drug treatment. However, their IOP was still not controlled below the target IOP level, which was generally determined by the stage of disease. However, as long as the IOP is controlled in a relatively safe range, even if the target IOP is not reached, we think there's no need for excessive surgical treatment. The more rapid the IOP elevated, the earlier the RNFL was damaged. INFRNFL and SUP-RNFL turned out to be the most IOPsensitive quadrants with the rate of RNFL change going almost in parallel with IOP levels. Meanwhile, the temporal superior sector seemed to be resistant to high IOP conditions until an RNFL loss of $\sim 20 \mu \mathrm{m}$ was detected in the temporal superior sector. It would help to track glaucoma progression not according to vision field but according to RNFL thickness, especially for patients who fail to finish the vision field examination like children or those with very poor eyesight. In addition, more and more attention has been paid to normal intraocular pressure glaucoma (NTG). Many patients still suffer from different degrees of RNFL injury, even though their intraocular pressure is controlled under $21 \mathrm{mmHg}$. The pathogenesis of NTG was investigated in some studies but still needs to be elucidated. The central retinal venous pressure first proposed by Baumann [25] in 1925 can be used to estimate intracranial pressure. In the 1970s, Ralph et al. [26]. found in the study of a monkey brain injury model that acute intracranial pressure increase can lead to increased IOP. Cerebrospinal Fluid Pressure (CSF-P) was found to be one of the factors influencing the optic nerve injury in glaucoma [27-31]. The RNFL damage pattern in NTG patients has yet to be studied. This study is mainly focused on the quantitative relationship between high IOP and RNFL. We hope to be able to develop an NTG animal model and conduct some related research in the future.

In addition, it was observed that besides high IOP, baseline/residual RNFL thickness was also important for RNFL thickness loss. C.K. Leung had reported in a five-year study of 64 glaucoma patients who had a greater baseline RNFL thickness was associated with an increased rate of reduction in RNFL thickness [8]. In this study, the IOPs of monkey models were always maintained at 40-50 $\mathrm{mmHg}$, the average RNFL thickness was from 97 to $75 \mu \mathrm{m}$ at a rate of $-21.86 \pm 3.10 \mu \mathrm{m} /$ week, and then it dropped to $65 \mu \mathrm{m}$ at a rate of $-4.00 \pm 1.70 \mu \mathrm{m} /$ week and finally decreased to $50 \mu \mathrm{m}$ at a rate of $-2.56 \pm 2.01 \mu \mathrm{m} /$ week. From the turning points of average RNFL thickness subsequent damage, it can be seen that the damage rate was gradually decreased with the reduction of the remaining RNFL thickness. The thinner the RNFL thickness is, the slower the damage rate becomes. It is difficult to observe the natural course of RNFL thickness loss under high IOP conditions in glaucoma patients because of the immediate treatment intervention once it is diagnosed. Since monkey/ COHT models have so many features that are similar to human beings/glaucoma patients, it is reasonable to believe they model the important effect of baseline/residual RNFL thickness on the rate of RNFL thickness loss.

Finally, OCT was demonstrated to be suitable for measuring RNFL thickness changes in a COHT monkey model. It is evident that in glaucoma eyes, the structural changes of the retina happen earlier than a detectable functional disorder using current examination approaches [32-35], among which OCT, as the most important device for measuring optic nerve structural damage, has become a widely used method for evaluating early stage glaucoma and disease progression [36, 37]. In previous reports, OCT has been shown to be a useful tool for detection of RNFL change in monkey models of glaucoma [15-17]. OCT detection of a monkey's RNFL, such as baseline RNFL thickness, the "ISNT" damage principle of RNFL, and "IOP-related RNFL loss" (the largest coefficient of association was the temporal inferior and superior sectors) were seen, which were similar to those of glaucoma patients. It was demonstrated that OCT was an ideal device to detect RNFL thickness change in COHT monkey models.

Regretfully, the relationship between 24-h IOP measurements and RNFL thickness loss was not included in this study. Further research will focus on this aspect.

In conclusion, the COHT monkey model was an ideal animal model that can be used for evaluating the relationship between IOP and RNFL thickness damage. Higher IOP was associated with faster rates of progressive RNFL loss. The level of IOP was a particularly vital factor for RNFL damage rate, and baseline/residual RNFL thickness was also important for RNFL subsequent damage. OCT was suitable for measuring the RNFL thickness change in COHT monkey models. These preliminary results might contribute to predicting the RNFL thickness loss trend and efficacy assessment of IOP-lowering therapies. 
Acknowledgements This work was supported by the National Natural Science Foundation of China (grant number: 81430009) and the Medical Scientific Research Foundation of Guangdong Province, China (grant number: A2018070).

\section{Compliance with ethical standards}

Conflict of interest The authors declare that they have no conflict of interest.

Publisher's note: Springer Nature remains neutral with regard to jurisdictional claims in published maps and institutional affiliations.

\section{References}

1. Bengtsson B, Heijl A. Diurnal IOP fluctuation: not an independent risk factor for glaucomatous visual field loss in high-risk ocular hypertension. Graefe's Arch Clin Exp Ophthalmol. 2005;243:513-8.

2. Guo L, Moss SE, Alexander RA, Ali RR, Fitzke FW, Cordeiro MF. Retinal ganglion cell apoptosis in glaucoma is related to intraocular pressure and IOP-induced effects on extracellular matrix. Invest Ophthalmol Vis Sci. 2005;46:175-82.

3. European Glaucoma Society. Terminology and guidelines for glaucoma. 4th edn. EU: European Union; 2014.

4. Gordon MO, Beiser JA, Brandt JD, Heuer DK, Higginbotham EJ, Johnson CA, et al. The ocular hypertension treatment study: baseline factors that predict the onset of primary open-angle glaucoma. Arch Ophthalmol. 2002;120:714-20. discussion829-30

5. Kass MA, Heuer DK, Higginbotham EJ, Johnson CA, Keltner JL, Miller JP, et al. The Ocular Hypertension Treatment Study: a randomized trial determines that topical ocular hypotensive medication delays or prevents the onset of primary open-angle glaucoma. Arch Ophthalmol. 2002;120:701-13. discussion 829-30

6. Heijl A, Leske MC, Bengtsson B, Hyman L, Bengtsson B, Hussein $\mathrm{M}$, et al. Reduction of intraocular pressure and glaucoma progression: results from the Early Manifest Glaucoma Trial. Arch Ophthalmol. 2002;120:1268-79.

7. Leske MC, Heijl A, Hussein M, Bengtsson B, Hyman L, Komaroff E, et al. Factors for glaucoma progression and the effect of treatment: the early manifest glaucoma trial. Arch Ophthalmol. 2003;121:48-56.

8. Leung CK, Cheung CY, Weinreb RN, Qiu K, Liu S, Li H, et al. Evaluation of retinal nerve fiber layer progression in glaucoma: a study on optical coherence tomography guided progression analysis. Invest Ophthalmol Vis Sci. 2010;51:217-22.

9. Leung CK, Yu M, Weinreb RN, Ye C, Liu S, Lai G, et al. Retinal nerve fiber layer imaging with spectral-domain optical coherence tomography: a prospective analysis of age-related loss. Ophthalmology. 2012;119:731-7.

10. Zhang X, Dastiridou A, Francis BA, Tan O, Varma R, Greenfield DS, et al. Baseline fourier-domain optical coherence tomography structural risk factors for visual field progression in the advanced imaging for glaucoma study. Am J Ophthalmol. 2016;172:94-103.

11. Noguchi T, Shimazawa M, Hamaguchi K, Araki T, Horai N, Hara $\mathrm{H}$. Relationship between elevated intraocular pressure and divided peripapillary sector retinal nerve fiber layer thickness in a cynomolgus monkey laser-induced ocular hypertension model. Ophthalmic Res. 2017;58:99-106.

12. Dwelle J, Liu S, Wang B, McElroy A, Ho D, Markey MK, et al. Thickness, phase retardation, birefringence, and reflectance of the retinal nerve fiber layer in normal and glaucomatous non-human primates. Invest Ophthalmol Vis Sci. 2012;53:4380-95.
13. Gardiner SK, Fortune B, Wang L, Downs JC, Burgoyne CF. Intraocular pressure magnitude and variability as predictors of rates of structural change in non-human primate experimental glaucoma. Exp Eye Res. 2012;103:1-8.

14. Shu Tu, Kang Li, Jingjing Huang, et al. Patterns of optic nerve head and retinal nerve fiber layer damage in the monkey chronic ocular hypertension model[J]. Chin J Optom Ophthalmol Vis Sci. 2014; $16: 436-40$.

15. Schuman JS, Pedut-Kloizman T, Pakter H, Wang N, Guedes V, Huang L, et al. Optical coherence tomography and histologic measurements of nerve fiber layer thickness in normal and glaucomatous monkey eyes. Invest Ophthalmol Vis Sci. 2007;48:3645-54.

16. Strouthidis NG, Grimm J, Williams GA, Cull GA, Wilson DJ, Burgoyne CF. A comparison of optic nerve head morphology viewed by spectral domain optical coherence tomography and by serial histology. Invest Ophthalmol Vis Sci. 2010;51:1464-74.

17. Zhang Z, Yang D, Sang J, Hou R, Liu K, Li Z, et al. Reproducibility of macular, retinal nerve fiber layer, and $\mathrm{ONH}$ measurements by OCT in Rhesus monkeys: The Beijing Intracranial and Intraocular Pressure (iCOP) Study. Invest Ophthalmol Vis Sci. 2012:53:4505-9.

18. Gaasterland D, Kupfer C. Experimental glaucoma in the rhesus monkey. Invest Ophthalmol. 1974;13:455-7.

19. Quigley HA, Hohman RM. Laser energy levels for trabecular meshwork damage in the primate eye. Invest Ophthalmol Vis Sci. 1983;24:1305-7.

20. Huang XR, Kong W, Qiao J. Response of the retinal nerve fiber layer reflectance and thickness to optic nerve crush. Invest Ophthalmol Vis Sci. 2018;59:2094-103.

21. Rajaei SM, Mood MA, Paryani MR, Williams DL. Effects of diurnal variation and anesthetic agents on intraocular pressure in Syrian hamsters (Mesocricetus auratus). Am J Vet Res. 2017;78:85-9.

22. Taniguchi T, Shimazawa M, Araie M, Tomita G, Sasaoka M, Kitazawa $\mathrm{Y}$, et al. Optic disc topographic parameters measured in the normal cynomolgus monkey by confocal scanning laser tomography. Br J Ophthalmol. 2005;89:1058-62.

23. Diniz-Filho A, Abe RY, Zangwill LM, Gracitelli CP, Weinreb RN, Girkin CA, et al. Association between intraocular pressure and rates of retinal nerve fiber layer loss measured by optical coherence tomography. Ophthalmology. 2016;123:2058-65.

24. Bowd C, Zangwill LM, Weinreb RN, Medeiros FA, Belghith A. Estimating optical coherence tomography structural measurement floors to improve detection of progression in advanced glaucoma. Am J Ophthalmol. 2017;175:37-44.

25. Baurmann M. Über die Entstehung und klinische Bedeutung des Netzhautvenenpulses. Dtsch Ophthalmol Ges. 1925;45:53-59.

26. Lehman RA, Krupin T, Podos SM. Experimental effect of intracranial hypertension upon intraocular pressure. J Neurosurg. 1972;36:60-6.

27. Volkov VV. [Essential element of the glaucomatous process neglected in clinical practice]. Oftalmol Zh. 1976;31:500-4.

28. Berdahl JP, Fautsch MP, Stinnett SS, Allingham RR. Intracranial pressure in primary open angle glaucoma, normal tension glaucoma, and ocular hypertension: a case-control study. Invest Ophthalmol Vis Sci. 2008;49:5412-8.

29. Berdahl JP, Allingham RR, Johnson DH. Cerebrospinal fluid pressure is decreased in primary open-angle glaucoma. Ophthalmology. 2008;115:763-8.

30. Jonas JB, Yang D, Wang N. Intracranial pressure and glaucoma. J Glaucoma. 2013;22(Suppl 5):S13-4.

31. Ren R, Wang N, Zhang X, Cui T, Jonas JB. Trans-lamina cribrosa pressure difference correlated with neuroretinal rim area in glaucoma. Graefe's Arch Clin Exp Ophthalmol. 2011; 249:1057-63.

32. Wollstein G, Schuman JS, Price LL, Aydin A, Stark PC, Hertzmark E, et al. Optical coherence tomography longitudinal 
evaluation of retinal nerve fiber layer thickness in glaucoma. Arch Ophthalmol. 2005;123:464-70.

33. Gracitelli CP, Abe RY, Tatham AJ, Rosen PN, Zangwill LM, Boer ER, et al. Association between progressive retinal nerve fiber layer loss and longitudinal change in quality of life in glaucoma. JAMA Ophthalmol. 2015;133:384-90.

34. Kuang TM, Zhang C, Zangwill LM, Weinreb RN, Medeiros FA. Estimating lead time gained by optical coherence tomography in detecting glaucoma before development of visual field defects. Ophthalmology. 2015;122:2002-9.
35. Sommer A, Katz J, Quigley HA, Miller NR, Robin AL, Richter $\mathrm{RC}$, et al. Clinically detectable nerve fiber atrophy precedes the onset of glaucomatous field loss. Arch Ophthalmol. 1991; 109:77-83.

36. Abe RY, Gracitelli CP, Medeiros FA. The use of spectral-domain optical coherence tomography to detect glaucoma progression. Open Ophthalmol J. 2015;9:78-88.

37. Meira-Freitas D, Lisboa R, Medeiros FA. Advances in the structural evaluation of glaucoma with optical coherence tomography. Curr Ophthalmol Rep. 2013;1:98-105. 